[Agr. Biol. Chem., Vol. 27, No. 3, p. 205 209, 1963]

\title{
Studies on the Polymorphism of L-Glutamic Acid
}

\section{Part VI. Separation of L-Glutamic Acid from the Fermentation Broth}

\author{
By Yoshiki Sakata and Kuniharu Takenochi \\ Ajinomoto Co., Inc., Kawasaki Factory, Kawasaki \\ Received October 25,1962
}

\begin{abstract}
The very minute crystals of L-glutamic acid of inferior properties were apt to be crystallized from the concentrated fermentation broth by the direct neutralization method, and it was clarificd from the results of the examination of the effects of concentration, temperature and residual sugar on the crystallization of L-glutamic acid from the broth that the production of such undesirable crystals was due to the crystallization of crystals in $\beta$-form. By ardling the amino acids which are effective on the crystallization of L-glutamic acid in $a$-form from the purified glutamate solution, the easily filtrable a-crystals could be obtained also from the concentrated broth by the direct neutralization method.
\end{abstract}

\section{INTRODUCTION}

Of the two alternative forms of L-glutamic acid crystal, the crystal in $a$-form is more preferable for the industrial handling such as sedimentation, filtration or separation, transportation and packaging in virtue of its granular shape and high velocity of crystal growth. Especially when L-glutamic acid was crystallized from the solution containing a plenty of impurity, the growth of $\beta$-crystal was much inhibited and crystals, which were so minute that the industrial separation of them was very difficult, were often obtained. The polymorphism of L-glutamic acid, therefore, is one of the most important factors in the MSG industry.

The authors applied the conception of the polymorphism of t-glutamic acid which was obtained from the results of the preceding reports ${ }^{1)}$, to the separation of L-glutamic acid from the fermentation broth, and the results are reported in this paper.

1) Y. Sakata, This Journal, 25, 829 (1961).

\section{EXPERIMENTAL AND DISCUSSION}

1. The Separation L-Glutamic Acid from the Broth by the Direct Neutralization Method.

L-Glutamic acid can be separated most simply from the broth by neutralizing the cell-free, concentrated broth with acid until the $\mathrm{pH}$ reaches the isoelectric point of L-glutamic acid. Chen and Huange) reported that the crystals thus obtained from the concentrated broth by the direct neutralization method were very minute and of low purity. On the other hand, they got the crystals of relatively high purity when Lglutamic acid was separated via the hydrochloride after the heat treatment of the broth with hydrochloric acid.

This experiment was carried out to examine the effects of some factors such as concentration and temperature on the crystallization of $\mathbf{L}$-glutamic acid from the broth by the direct neutralization method, without taking the yield into consideration.

(1) Materials.

L-Glutamic acid fermentation broth: Fermented with Brevibacterium lactofermentum No. 2256. The yield of L-glutamic acid to the initial sugar was $42.0 \%$. The residual sugar was $7 \%$ of initial one. $\mathrm{pH} 8$.

Hydrochloric acid: $35 \%$, commercial material of reagent grade.

2) Chen and Huang, J. Ferm. Technol., 37, 322 (1959). 
(2) Effect of the Concentration.

The broth was concentrated under reduced pressure to the concentration of L-glutamic acid from $8 \%$ to $20 \%$, after cells were filtered out using fuller's earth as filter aid.

To $500 \mathrm{ml}$ beakers, $300 \mathrm{ml}$ of each concentrated broth was taken respectively, and warmed to about $50^{\circ} \mathrm{C}$. Then hydrochloric acid was added with stirring until pH 3.2 was reached, taking about 30 minutes for crystallization of L-glutamic acid. The slurry was cooled to room temperature with further stirring and was left for standing for 1 day at room temperature. The crystals were filtered with a Buchner's funnel, and washed twice with water equiamount to the wet cake. The content of L-glutamic acid in the wet cake was determined by the enzymatic method, using Lglutamic acid decarboxylase, and the loss on drying at $90^{\circ} \mathrm{C}$ was also measured. The crystal form of the L-glutamic acid obtained was decided by the powder $\mathrm{X}$-ray method. The result is shown in Table $\mathrm{I}$.

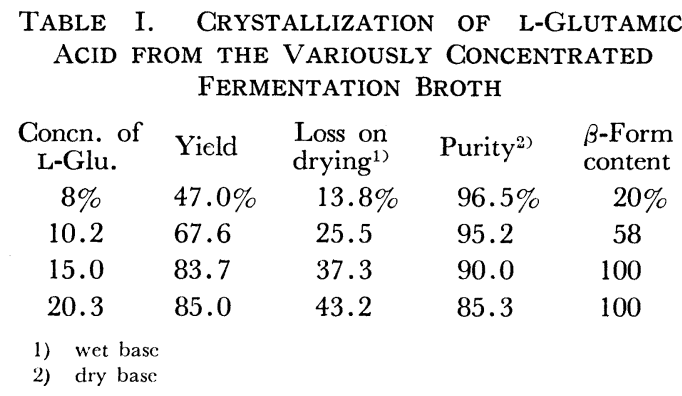

As seen obviously in the result, although the yield increased correspondingly in accordance with the increase of the concentration, on the contrary the filtrability and the purity of the crystals obtained became worse. This low filtrability was due to the $\beta$-crystal formation, judging from the result of the $\mathrm{X}$-ray analysis. As a $\beta$-crystal has needle-like or flaky shape and its sedimenting and filtrating properties are not so good even when it is crystallized from the purified solution, it can be readily supposed that $\beta$-crystals will deposit as very minute crystals from its impure solution due to the inhibitory effect of co-existing impurities on the crystal growth.

\section{(3) Effect of Temperature.}

Generally speaking, the higher the temperature, the higher the velocity of crystal growth, thus it will be preferable for getting large crystals that crystallization is carried out at temperature as high as possible.
Therefore, temperature should be regarded as one of the most important factors concerning the industrial crystallization.

The concentrated broth containing $15.0 \%$ of $\mathrm{L}$ glutamic acid was prepared by the same procedure as (2). Hydrochloric acid was added with constant stirring to crystallize L-glutamic acid at temperatures from $10^{\circ} \mathrm{C}$ to $60^{\circ} \mathrm{C}$, and the effect of temperature on the crystallization was observed. The experimental result was shown in Tables II and III.

\section{TABle II. GRYSTAllization OF L-Glutamic ACID FROM THE CONCENTRATED FER- MENTATION BROTH AT VARIOUS TEM- PERATURES}

\begin{tabular}{lccl} 
Temp. & $\begin{array}{c}\text { Yield of } \\
\text { Glu. }\end{array}$ & \multicolumn{2}{c}{ Obtained Crystals } \\
\cline { 3 - 4 } $10^{\circ} \mathrm{G}$ & $83.5 \%$ & $19.80 \%$ & $95.0 \%$ \\
20 & - & 15.03 & 94.8 \\
30 & 83.0 & 18.32 & 95.5 \\
40 & - & 30.8 & 92.3 \\
50 & - & 38.0 & 90.8 \\
60 & 82.7 & 37.2 & 90.7
\end{tabular}

TABle III. OBSERVATION OF THE CRYStals OBTAINED IN ExP. 3.

\begin{tabular}{cl} 
Temp. & \multicolumn{1}{c}{ Shapes of the crystals } \\
$10^{\circ} \mathrm{C}$ & granular, mostly $\alpha$-form \\
20 & granular, mostly $\alpha$-form \\
30 & mostly $\alpha$-form, a little quantity of $\beta$ \\
40 & $\alpha$ and $\beta$ half and half \\
50 & minute, flaky, mostly $\beta$-form \\
60 & minute, flaky, $\beta$-form
\end{tabular}

Contrary to the usual case, the higher the temperature, the less desirable the state of crystals obtained in the case of the fermentation broth, as shown in the result. The cause of this unexpected result could be clarified upon observation of the crystals obtained under a microscope as seen in Table III. That is, at relatively low temperature, mostly $a$-crystals were obtained; on the other hand, at higher temperature than $30^{\circ} \mathrm{C}, \beta$-crystals deposited and they became very minute as the temperature rose higher. It was reported previously that the tendency for $\beta$-crystals to crystallize was seen as the temperature rose. Although there is a considerable difference as to the crystal growth, it may safely be said that the crystallization of $L$ glutamic acid from the broth resembles closely with that from the purified glutamate solution. 


\section{Effect of Some Sugars on the Polymorphic Crystal- lization of L-Glutamic Acid.}

The cell-free, concentrated broth contains various impurities such as residual sugar, amino acids, organic acids etc. Among these, the content of residual sugar occupies the major portion of impurities and it comes to about $17 \%$ of $\mathrm{L}$-glutamic acik; on the other hand, the contents of amino acids and organic acid will be so little that they affect slightly on the crystallization of L-glutamic acid. Besides, an amount of protein will be contained, caused by the autolysis of cells, and these colloidal substances will affect much on the crystal growth.

The experimental results showed that for getting the easily filtrable crystals it is essential to crystallize L-glutamic acid in $a$-form; the following experiment was carried out to examine the effect of some sugars, the representative impurity in the broth, on the polymorphic crystallization of L-glutamic acid.

\section{(1) Materials.}

Mono-sodium L-glutamate monohydrate: commercial material. N:7. $48 \%$. $[\alpha]_{\mathrm{D}}^{20} 25.12(2 \mathrm{~N} \mathrm{HCl})$.

Glucose, fructose, galactose, sucrose, lactose: commercial materials of reagent grade. Hydrochloric acid: $35 \%$ commercial reagent.

\section{(2) Experimental.}

To the solutions warmed to about $40^{\circ} \mathrm{C}$ and containing $14.5 \%$ of mono-sodium L-glutamate monohydrate, 1 to $5 \%$ of either glucose, fructose, galactose, sucrose or lactose were added and dissolved, respectively, and then hydrochloric acid was added with constant stirring until the $\mathrm{pH}$ reached 3.2. The crystallized L-glutamic acid was inspected under a microscope, and the crystal form was judged from their shape.

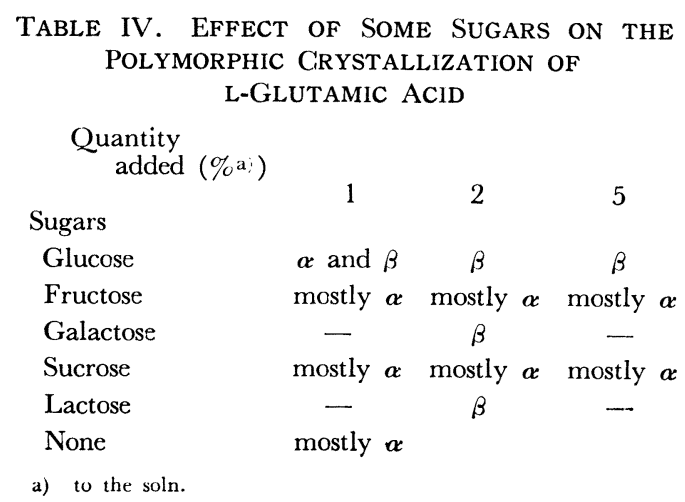

The result of observation is shown in Table IV. As seen from the result in Table IV, L-glutamic acid is apt to crystallize in its $\beta$-form under the coexistence of glucose or galactose or lactose. And the other sugars, i.e., fructose and sucrose did not show any effect on the polymorphic crystallization of $\mathrm{L}$ glutamic acid. This result indicates that only aldose shows the $\beta$-form crystallizing effect.

\section{Effect of the Addition of Amino Acids on the Separability of L-Glutamic Acid by the Neutraliza- tion Method. \\ From the results obtained in each experiment de-} scribed above, an opinion regarding the crystallization of L-glutamic acid from the fermentation broth has been deduced as follows: As already clarified in the preceding paper, the $\beta$-crystal is more stable than the $a$-crystal in the aqueous solution, and $\mathrm{L}$-glutamic acid is apt to be crystallized in its $\beta$-form, when it is separated from the aqueous solution. In the case of the separation of L-glutamic acid from the broth, it is most likely that the crystals in $\beta$-form are also obtained, because few impurities which have an inhibitory effect on the crystallization of $\beta$-crystals are contained in the broth. Furthermore, the residual sugar contained in the broth will render L-glutamic acid to be crystallized more easily in $\beta$-form. As the growth of $\beta$-crystals separated from the broth is likely inhibited considerably by impurities co-existing in the broth, the filtrability of the crystals, even of the purified ones, will become worse. Therefore, it may be said that the pursuit of the optimum condition for the separation of L-glutamic acid from the fermentation broth is just replaced by the pursuit of the optimum condition to crystallize the $a$-crystals from the broth. As for the condition to get the $a$-crystals from the aqueous solution, it was already clarified from the experimental results reported in the preceding papers that the lower the temperature and the concentration of glutamate solution, the more easily the $a$-crystals were obtained and also that under the co-existence of some amino acids such as L-phenylalanine, L-cystine, L-leucine, L-aspartic acid and $\mathrm{L}$ tyrosine, L-glutamic acid in $a$-form was easily crystallized. The result of the experiment-1 suggests that the conception of the polymorphism of L-glutamic acid obtained for the purified one can be applied also to the fermentation broth. As previously reported, when L-glutamic acid is separated from the protein hydrolyzate, $a$-crystals are crystallized and this is due 
to the co-existence of many and much amino acids, and they can be relatively easily filtered. These facts show that the crystallization of $\mathrm{I}$-glutamic acid from the broth is rather similar to that from the purified glutamate solution from a view-point of the polymorphic crystallization. The production of very minute and infiltrable $\beta$-crystals from the broth seems to be caused by lack of co-existing amino acids. Therefore, the crystallization of a-crystals may be expected by adding the amino acids which are effective on the crystallization of $a$-crystals to the fermentation broth.

The following experiments were carried out according to the suggestion described above, using Lphenylalanine and amino acids mixture as additions.

\section{(1) Materials.}

Concentrated broth: The concentrated broths containing from $10 \%$ to $25 \%$ of $\mathrm{L}$-glutamic acid were prepared by the same procedure as described in 1 .

L-Phenylalanine: commercial material of reagent grade.

Mother liquor of crude L-glutamic acid hydrochloride: The mother liquor of the crude crystals of Lglutamic acid hydrochloride which was separated from the hydrochloric acid hydrolyzate of wheat gluten.

$$
\begin{aligned}
& \text { L-Glu. } 1.57 \% \text {, L-Leu. } 2.70 \% \text {, L-Phe. } 1.10 \% \text {, } \\
& \text { L-Cys. } 0.80 \%
\end{aligned}
$$

Solution of crude L-glutamic acid hydrochloride:

The aqueous solution of crude L-glutamic acid hydrochloride which was separated from the hydrochloric acid hydrolyzate of soybean meal.

L-Glu. $15.0 \%$, L-Cys. $0.07 \%$, L-Leu. $0.8 \%$,

L-Phe. $0.5 \%$, L-Tyr. $0.5 \%$

Hydrochloric acid: $35 \%$ commercial material of reagent grade.

\section{(2) Experiment A.}

To each concentrated broth containing from $10 \%$ to $25 \%$ of L-glutamic acid, $0.5 \%$ of $\mathrm{L}$-phenylalanine was added and warmed to about $50^{\circ} \mathrm{C}$; and then hydrochloric acid was added gently until the $\mathrm{pH} 3.2$ was reached with constant stirring to crystallize $L_{-}$glutamic acid. After the slurries were cooled to room temperature and left for standing for 1 day at room temperature, the crystals were filtered with a Buchner's funnel and washed twice with water equiamount to the wet cake each time. The same crystallizing procedures were carried out without addition of $\mathrm{L}^{-}$ phenylalanine. Loss on drying at $90^{\circ} \mathrm{C}$ and content of L-glutamic acid of the obtained crystals were determined, where the determination of L-glutamic acid was executed by the enzymatic method, using Lglutamic acid decarboxylase. The experimental result is shown in Table $\mathrm{V}$.

\section{TABLE V. EFFECT OF ADDITION OF L-PHENYL-}

\begin{tabular}{|c|c|c|c|c|}
\hline \multirow{2}{*}{$\begin{array}{l}\text { Concn. } \\
\text { of Glu. }\end{array}$} & \multicolumn{2}{|c|}{$0.5 \%$ L-Phe. added } & \multicolumn{2}{|c|}{ Control } \\
\hline & $\begin{array}{c}\text { Loss on } \\
\text { drying }\end{array}$ & Purity & $\begin{array}{c}\text { Loss on } \\
\text { drying }\end{array}$ & Purity \\
\hline $9.7 \%$ & $12.0 \%$ & $96.3 \%$ & $32.0 \%$ & $92.5 \%$ \\
\hline 14.6 & 12.7 & 96.5 & 37.9 & 86.3 \\
\hline 18.9 & 21.5 & 95.5 & 42.7 & 84.7 \\
\hline 24.5 & 33.4 & 90.8 & 50.5 & 80.2 \\
\hline
\end{tabular}
ALANINE

As seen obviously in Table $\mathrm{V}$, the filtrability of the obtained crystals could be much improved by adding L-phenylalanine, and the crystals of considerably higher purity than the control were obtained. Needless to say, this is attributed to the $a$-form crystallizing effect of the added L-phenylalanine. Practically considering, it will be more economical to use amino acids mixture containing the amino acids which are especially effective on the crystallization of $a$-crystals, instead of the purified materials. Using the amino acids solution which can be easily obtained in the process of the separation of L-glutamic acid from the hydrolyzate of wheat gluten, soy bean meal or other vegetable proteins, the authors carricd out the same experiments as $\mathbf{A}$, and the procedures and the results are described in the following.

\section{(3) Experiment B.}

To $1 \mathrm{~kg}$ of the concentrated broth containing $20.7 \%$ of L-glutamic acid, $100 \mathrm{~g}$ of the mother liquor of crude L-glutamic acid hydrochloride was added and after the mixture was warmed to about $50^{\circ} \mathrm{C}$, hydrochloric acid was added gently until $\mathrm{pH} 3.2$ was reached with constant stirring to crystallize L-glutamic acid. After the slurry was cooled to room temperature with stirring and left to stand for 1 day at room temperature, the crystals were separated with a Buchner's funnel, and washed with $300 \mathrm{ml}$ of water, and $219 \mathrm{~g}$ of the wet crude crystals was obtained. The loss on drying at $90^{\circ} \mathrm{C}$ was $16.2 \%$ and the purity on dry base was $94.8 \%$. The yield of L-glutamic acid was $84.0 \%$.

The same procedure was carried out, without addition of the mother liquor of crude L-glutamic acid hydrochloride. The very minute, undesirable crystals in $\beta$-form were obtained, and the filtration and the washing of them were difficult. The wet, crude 
crystals $(348 \mathrm{~g})$, the loss of which on drying was $40.5 \%$, and the purity of which on dry base was $84.5 \%$, was otbained. The yield of L-glutamic acid was $84.6 \%$.

\section{(4) Experiment C.}

To $1 \mathrm{~kg}$ of the concentrated broth containing $18.5 \%$ of L-glutamic acid which was warmed to about $50^{\circ} \mathrm{C}$, $850 \mathrm{~g}$ of the solution of crude 1 -glutamic acid hydrochloride was added gently with constant stirring until $\mathrm{pH} 3.2$ was reached to crystallize L-glutamic acid. After the slurry was cooled to room temperature with stirring, and left to stand for 2 days, the crystals were separated with a Buchner's funnel, and washed with $400 \mathrm{ml}$ of water.

Operations of the filtration and the washing were relatively easy, and $300 \mathrm{~g}$ of the crude crystals, the loss of which on drying was $13.5 \%$ and the purity of which on dry base was $95.2 \%$ and which contained $12.5 \%$ of $\beta$-form (by X-ray analysis) was obtained.
The yield of L-glutamic acid was $80 \%$.

As seen from the results of a series of the experiments described above, the $a$-crystals which could be easily filtered were crystallized from the concentrated fermentation broth by addition of amino acids.

The crystallization and separation of L-glutamic acid is one of the most important processes in the MSG inclustry, and it has been shown in this paper how usefully the fundamental knowledge of the polymorphism of L-glutamic acid can be applied in the practical process of the MSG industry.

Acknowledgement. The authors wish to express their sincere thanks to Mr. S. Inagawa, Managing Director of Ajinomoto Co., Inc. and Superintendent of Kawasaki Factory for his encouragement, and to Mr. N. Nagashima for his analysis by X-ray method. 